\title{
LP based solution for Security Constrained Optimal Power Flow including Power Routers
}

\author{
D.HARINI, M.RAMESH BABU, C.VENKATESH KUMAR \\ Department of Electrical and Electronics Engineering \\ St. Joseph's College of Engineering \\ OMR, Chennai, Tamilnadu, INDIA
}

\begin{abstract}
Power Routers offers many benefits to the power system, it helps in improving the existing transmission asset utilization. Security Constrained Optimal Power Flow (SCOPF) is becoming more important in the electrical power system especially in the present deregulated environment. This paper focuses on completely linearizing the complex non-linear SCOPF problem. The objective function is linearized using Piecewise linearization technique and the constraints are framed using linear sensitivity factors. A formal extension is made to the traditional SCOPF by including power router control in the post contingency time frame. DC power flow analysis is used to calculate the real power flow in the lines.Thus, in this paper, further minimization of cost is achieved by using Power Router control and it is compared with the conventional SCOPF.
\end{abstract}

Key-Words: - Power Routers, Security Constrained Optimal Power Flow, Linear Programming, Sensitivity factors.

Received: May 5, 2019, 2020. Revised: December 22, 2020. Accepted: January 12, 2020.

Published: February 5, 2020.

\section{Introduction}

The reliable and secure operation of electrical infrastructure which is responsible for providing electricity for the most essential services of modern society is of utmost importance in the operation and development of today's electrified world. In a power system, Security is defined as the ability of the power system to operate within its safe limits and remain stable even if unexpected failures or contingency occurs [1]. SCOPF problem is solved to obtain minimum fuel cost and by ensuring that the power system operates securely even under contingency conditions.

The increase in energy demand and the integration of Renewable Energy Sources (RESs) are stressing the power grid, and also prompt the system operators to take control measures for managing the power flow more efficiently and intelligently. PRs increase transfer capabilities of the power grid by enabling active routing of power flows in a power system. An extension to the conventional SCOPF is made in this paper that will increase the benefit of using PRs as a means of transmission expansion and increased asset utilization. This is achieved by modifying the constraints used for optimization to incorporate power routing capabilities during the precontingency and post-contingency time frames.
In 1974, Podmore [2] introduced the concept of linear security constraints and solved the optimization problem using gradient projection method. In the same year Wollenb0erg and Stadlin [3] used linearized network model, and using the security constraints developed a security constrained economic dispatch algorithm. Linear programming techniques were also applied to the Security Dispatch problems in the 1970's [4]. In 1987, Sanders and Momroe [5] presented a real-time constrained economic dispatch calculation algorithm, which is called as the CEDC and described a Newton-Raphson based linearized constraint approach in the context of active power security control. P.W. Sauer [6] introduced the mathematical formulation of power distribution factors.

The SCOPF algorithm determines the power output from each generator, and other controllable quantities (e.g., area interchange), such that even if an outage occurs the power system continue to operate securely without violating the limits. The set of contingencies that are used in the SCOPF algorithm are chosen by the system operators based on the history of the power system, their operational experience and system planning.

Contingency analysis is done using sensitivity factors, such as Power Transfer Distribution Factor 
(PTDF) and Outage Transfer Distribution Factor (OTDF), where PTDFs are used to frame the constraints for the optimization problem in the precontingency time frame and OTDFs are used to frame the constraints in the post-contingency time frame.

In this paper, a formal extension is made to the conventional Security Constrained Optimal Power Flow problem by including power flow router control in the pre contingency and post contingency timeframe. The extended SCOPF is formulated with a single objective function, minimization of total fuel cost and security constraints are also included to ensure system's stability in the post-contingency case and dynamic power flow control in the lines is achieved by including Power Flow Router (PFR). The linear programming based technique is used to solve the extended SCOPF problem.

\section{Concept of Power Routers}

Power electronics based power flow routers provide an easy and economic control of power flows in the electricity network. By adding additional controls, fast power flow routing can increase the security operational range of bulk power systems, both under normal operating conditions and during the occurrence of contingencies. Increased integration of power flow

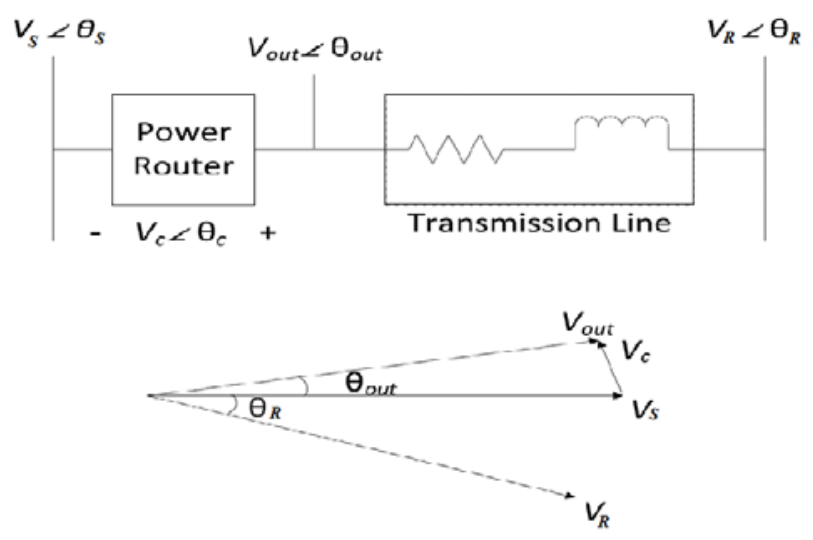

Fig.1. Power flow router injecting voltage magnitude and phase angle

routers could allow the large power system to operate at a lower cost and also increases the level of security. In this work, power flow router sensitivities have been developed which enables corrective flow routing capabilities during postcontingency conditions.
Phase Shifting Transformers (PST) are useful means of control of active power flow [11], but it is used only for static applications. Since PST is a mechanical device, it is slow in operation compared to power electronic based devices. Flexible AC Transmission System (FACTS) devices are used for a variety of purposes, including voltage regulation, controlling power flows, reducing harmonics, and VAR compensation [12]. Unified Power Flow Controller (UPFC) is a FACTS based device and has the capability of regulating the power flow and minimizing the power losses simultaneously [13]. But the disadvantage of FACTS devices is that they are quite expensive for high quality semi-conductor materials are required.

Some new technologies have been introduced to control the power flow in the transmission lines which operates at low cost compared to FACTS devices. It includes fractionally rated Back-To-Back (FR-BTB) converter and Controllable Network Transformer (CNT). The FR - BTB converter has some features like fractional converter rating, reliability and scalability, thus making it a right option for the dynamic control of power flow in meshed systems [14]. CNT can be realized by augmenting Load Tap Changer (LTC) with small fractionally rated bidirectional direct $\mathrm{AC}-\mathrm{AC}$ converters [15]. The use of Dual Virtual Quadrature Sources (DVQS) scheme allows the CNT to control both the magnitude as well as the phase angle of the bus voltage, which enables it to achieve vernier control over the power flow in a meshed network [16].

Most power-electronics based power routers inject a voltage in series with transmission lines to effectively change the impedance of the line and thus changes the flow of power throughout the power system. Figure.1 represents a power router placed on a transmission line between bus 1 and bus 2 . The power flow router creates a voltage phasor $\left(\mathrm{V}_{\mathrm{c}}\right)$ that manipulates the sending bus voltage phasor $\left(V_{s}\right)$ to create a new output voltage phasor $\left(V_{r}\right)$. This output voltage phasor is now the effective voltage phasor of the sending bus, but only for this specific transmission line with a power flow router present. Because the output voltage phasor can be controlled, the voltage difference across the transmission line and, thus, the amount of power that flows through the transmission line are also controlled.

The power electronic based devices FR-BTB and CNT can control both voltage magnitude and the voltage phase angle of the bus. They have a fast 
response and are more economical compared to the conventional power flow controlling devices. In this work, the integration of Power routers in the conventional SCOPF problem is done, which further minimizes the operating cost of the system. Equation (1) shows the real power flow along a normal transmission line. The power flow through a line is a function of 4 variables:

- Sending end voltage phase angle $\left(\delta_{\mathrm{s}}\right)$

- Receiving end voltage phase angle $\left(\delta_{\mathrm{r}}\right)$

- Sending end voltage magnitude $\left(\mathrm{V}_{\mathrm{s}}\right)$

- Receiving end voltage magnitude $\left(\mathrm{V}_{\mathrm{r}}\right)$

$P_{s r}=V_{s}^{2} G_{s r}-V_{s} V_{r}\left[G_{s r} \cos \left(\delta_{s}-\delta_{r}\right)+B_{s r} \sin \left(\delta_{s}-\delta_{r}\right)\right]$

$P_{s r}=\frac{V_{s}^{2} G_{s r}}{t_{s r}^{2}}-\frac{V_{s} V_{r}}{t_{s r}}\left[G_{s r} \cos \left(\delta_{s}-\delta_{r}-\phi_{s r}\right)+B_{s r} \sin \left(\delta_{s}-\delta_{r}-\phi_{s r}\right)\right]$

When a power router is inserted in series with an existing transmission line the equation for the real power flow changes to that in equation (2), which introduces the injected voltage phase ( $\Phi$ sr) and a tap ratio (tsr) which is dependent on the voltage magnitude injected by the power flow router.

In the present work, only a voltage phase injection will be considered. This is so that we can focus on the routing of real power in power systems. Routing of real power is of major concern because it is the portion of power that is bought and sold in energy markets. The voltage angle has more influence on the real power flows in a power system.

\section{Security-Constrained Optimal Power Flow}

Secure operation of the power system refers to the capability of the system to withstand unexpected failures and disconnection of components, and continues to operate safely, supplying uninterrupted power to the consumers [1]. Security constraints are not taken into account in the classical OPF formulation. SCOPF can be defined as the programs that can make certain adjustments to the control variables in the pre-contingency case to avoid violations during post-contingency conditions, thus new "security constraints" or "contingency constraints" are included in the classical OPF problem.

The algorithm for SCOPF solution is shown in Fig.2 [4] starts with solving an OPF with ( $\mathrm{N}-0)$ constraints only. This dispatch will satisfy generation and all line flow limits, but if any transmission line outage occurs, then the lines would get overloaded. The optimization loop begins by solving the DC power flow, which gives the MW power flow in the lines. This information is then used to calculate the sensitivities which are used to form the pre-contingency and post-contingency constraints for the optimization problem. The problem is then solved by Linear Programming (LP) technique which gives the generator real power set points at the lowest cost of operation satisfying all the constraints. The process is repeated until the error value is less than the threshold range. The error is the difference between the generator set points and the previous iteration solution.

\section{Contingency Analysis}

Many of the problems that occur on a power system can cause serious trouble within a short period of time that the operator cannot take immediate action once the failure has occurred. Hence the modern computers are equipped with programs for contingency analysis, such that these programs could model possible system troubles before they arise. The usual contingencies that occur in the power system are Generator outage or Transmission line outage. These outages may lead to line limit violations [9].

The problem of studying thousands of possible outages is very difficult to solve. The results cannot be presented quickly. Hence the easiest way of providing a quick calculation of possible overloads is by using linear sensitivity factors. These factors are derived from DC load flow and it shows the approximate change in line flows for any changes in the generation.

\section{Distribution Factors}

Sensitivities of power systems are used to account for the transmission line constraints during both pre-contingency and post-contingency conditions. Two commonly used sensitivities are Power Transfer Distribution Factor (PTDF) and Outage Transfer Distribution Factor (OTDF). PTDFs are used to account for transmission line constraints in the pre-contingency case. OTDFs are used to account for transmission line constraints in the post-contingency time frame to ensure system security in the event of failure of a line or a generator connected to the system. 


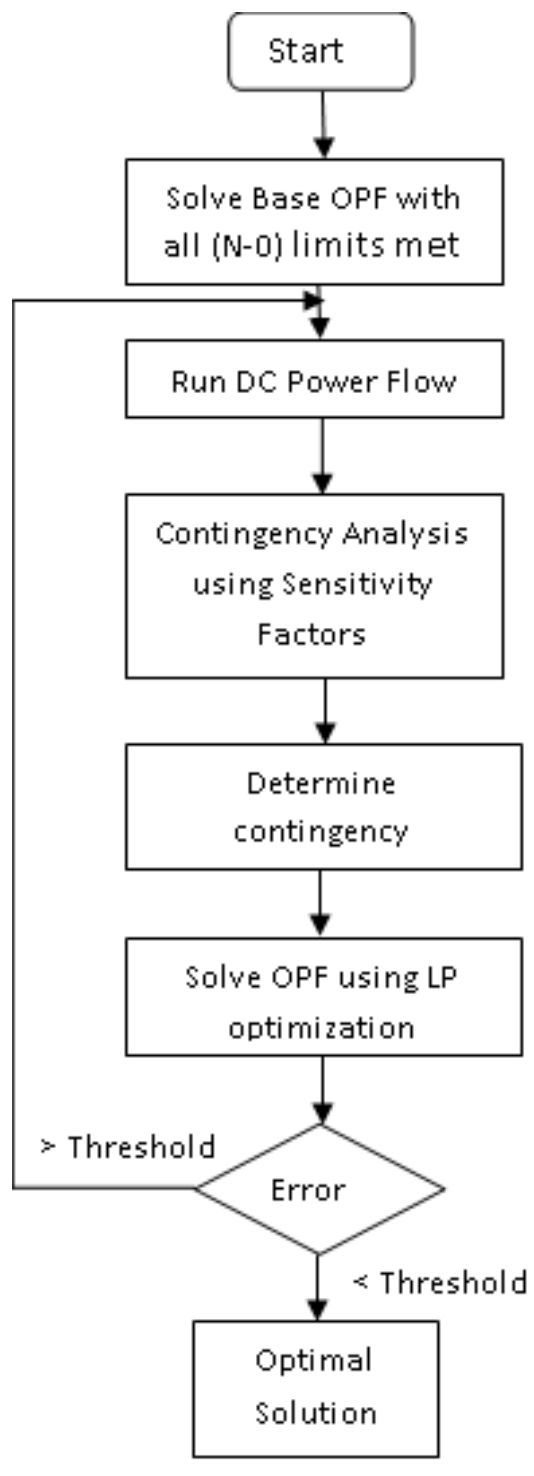

Fig.2. Algorithm for SCOPF solution methodology

\subsection{PTDF}

PTDFs are calculated using DC power flow analysis with nominal loss in accuracy. It estimates how the power flows with the lines distribute in the pre-contingency time frame. In this paper, the factors are computed by using DC power flow equations to simplify the calculations. Also, since the main focus of this paper is on the real power flows, the information lost in reactive power flow is acceptable.

The PTDF factor represents the sensitivity of the flow on line 1 to a shift of power from bus $i$ to $j$.

$$
P T D F_{i, j, l}=\frac{\Delta f_{l}}{\Delta P}
$$

Thus the new power flow fl,new on each line 1 in the network could be calculated using a precalculated set of "PTDF" factors as follows:

$$
f_{l, \text { new }}=f_{l}^{0}+P T D F_{i, j, l} \Delta P
$$

Here, fl0 represents the flow on line 1 before the outage of either another line or a generating unit.

\subsection{OTDF}

The Outage Transfer Distribution Factor (OTDF) is used to estimate how the power flows in the line distribute in the post-contingency time frame. To calculate OTDF, an intermediate factor called Line Outage Distribution Factor (LODF) is needed to be calculated. LODF estimates how the power flows in the lines redistribute when transmission circuits are lost. By definition, LODF has the following meaning:

$$
L O D F_{l, k}=\frac{\Delta f_{l}}{f_{k}^{0}}
$$

Here, $f_{k}^{0}$ represents the original flow on line $k$ before it was outaged (opened) and $\Delta f_{l}$ represents the change in real power on line 1 due to outage of line $k$. Thus the OTDF is the combination of both PTDF and LODF.

$$
O T D F_{l, k}=P T D F_{l, i}+\left(P T D F_{k, i} \times L O D F_{l, k}\right)
$$

The first term PTDF accounts for the power flow in the lines in the pre-contingency case. The second term accounts for the power flows during the postcontingency case. Thus OTDF tells us how the generators directly affect the power flow in the lines when a contingency occurs and this factor can be used to set constraints for the generators to avoid overloading of lines.

\subsection{Power Flow Router Sensitivities}

To enable analysis of the impact of power flow routers on the power system as corrective control, sensitivity is needed. We name this sensitivity the Power Flow Routing Distribution Factor (PFRDF). This sensitivity calculates how much the power flow in each branch of the power system will change with respect to a phase angle injection on a transmission line.

The PFRDF is defined as a sensitivity relating the power flow through a transmission line with respect to a PR phase injection. When a voltage phase is injected along a transmission line the state 
variables (i.e., voltage angles and magnitudes) of the power system will all change. This needs to be factored into the sensitivity along with the actual change in voltage phase injected, if the transmission line has a power flow router. The change in state variables can be calculated by multiplying the power flow Jacobian with the partial derivative of power injection of each bus with respect to the voltage phase injected into the power system given in equation (7). This product represents how the state variables of the power system change with respect to a phase injection by the power router in the power system.

$$
\left[\begin{array}{c}
\frac{\partial \delta_{i}}{\partial \phi_{x y}} \\
\frac{\partial \delta_{j}}{\partial \phi_{x y}}
\end{array}\right]=\left[\begin{array}{llll}
\frac{\partial \delta_{i}}{\partial P_{1}} & \frac{\partial \delta_{i}}{\partial P_{2}} & \cdots & \frac{\partial \delta_{i}}{\partial P_{n}} \\
\frac{\partial \delta_{j}}{\partial P_{1}} & \frac{\partial \delta_{j}}{\partial P_{2}} & \cdots & \frac{\partial \delta_{j}}{\partial P_{n}}
\end{array}\right]\left[\begin{array}{c}
\frac{\partial P_{1}}{\partial \phi_{x y}} \\
\vdots \\
\frac{\partial P_{n}}{\partial \phi_{x y}}
\end{array}\right]
$$

The change in power injection will occur only at the two buses of a transmission line to which a power router is connected. These changes in power injections can be seen as the power router simulating additional load or generation. The relationship between a phase angle injected and the state variables of a specific transmission line given in equation (7) can now be multiplied by the transmission line derivatives. This result in the first term of (8), gives the real power change in any branch with respect to how the line's state variables have changed. In most of the lines in the system this term is enough to show how much the real power has changed. However, the first term of the equation (8) does not account for the case where the power flow router is located on the line of interest. Because of this, an additional term needs to be added to the first term to account for how the power flow router is controlling power along that specific branch with the power flow router present. The second term is non-zero only when a power flow router is present on the branch of interest.

$$
\operatorname{PFRDF}_{i j, \phi_{x y}}=\left[\begin{array}{ll}
\frac{\partial P_{i j}}{\partial \delta_{i}} & \frac{\partial P_{i j}}{\partial \delta_{j}}
\end{array}\right]\left[\begin{array}{l}
\frac{\partial \delta_{i}}{\partial \phi_{x y}} \\
\frac{\partial \delta_{j}}{\partial \phi_{x y}}
\end{array}\right]+\frac{\partial P_{i j}}{\partial \phi_{x y}}
$$

This PFRDF now gives the user a value of how much real power, in p.u., is flowing on an arbitrary branch in the power system with respect to a phase angle injection, in radians, from one power flow router in the power system. There is the possibility of expanding this equation to accommodate multiple power flow routers in the power system.

There are two components affecting the power flow in branches, the power transfer and the power flow router. The power transfer component can be represented by the PTDF. The power flow router component is represented by the PFRDF in actual MW units. These two components are summed together to create the Power Flow Router Power Transfer Distribution Factor (PFRPTDF) seen in equation (9).

$$
\operatorname{PFRPTDF}_{m n, i, \phi_{x y}}=P T D F_{m n, i}+P^{P R D F_{m n, \phi_{x y}}}
$$

In the case of an outage the regular PFRDF cannot be used. Instead a Power Flow Router Outage Distribution Factor (PFRODF) is used, which represents how power flows redistribute in a power system with a line outage. The normal PFRDF must only be modified to account for the removal of a branch. This is done by modifying the power flow Jacobian in (7) to effectively remove the outaged branch. The normal PFRDF calculation, can then be carried out with the modified Jacobian to produce a PFRODF. The PFRODF enables the power flow router to be used as correction capability during post-contingency situations. The two components, the PFRODF and the original OTDF, are then summed together to form the PFROTDF given in equation (10).

$$
\mathrm{PFROTDF}_{m n, l k, \phi_{x y}}=O T D F_{m n, l k}+P F R O D F_{m n, l k, \phi_{x y}}
$$

The effects of the Power Router (PR) on the power system are independent of other power transfers and other PRs. In this work, only a single $\mathrm{PR}$ is considered for one particular line.

\section{Problem Formulation}

The Security Constrained Optimal Power Flow optimization problem is solved using Linear Programming Technique, in which both the objective function and the constraints are in linearized form.

\subsection{Piecewise Linearization of Objective Function}

The objective function considered here is the minimization of total fuel cost. The fuel cost curve of a power system is a non-linear curve and the corresponding fuel cost function is also given in a 
non-linear form. The non-linear fuel cost function is linearized by using the Piecewise linearization technique.

We express the nonlinear input-output or cost functions as a set of linear functions. The nonlinear cost function is:

$$
F_{i}\left(P_{i}\right)=a_{i}\left(P_{i}^{2}\right)+b_{i}\left(P_{i}\right)+c i(\$ / \mathrm{hr})
$$

The nonlinear curve is divided into three segments, where the three segments will be represented as $P_{i 1}, P_{i 2}, P_{i 3}$. Each segment power is measured from the start of the $\mathrm{x}$ segment.

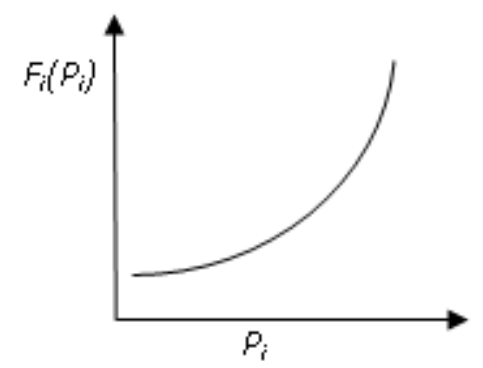

Fig.3. Non-linear fuel-cost curve

Each segment has a slope designated $\mathrm{s}_{\mathrm{i} 1}, \mathrm{~s}_{\mathrm{i} 2}$ and si3 and then the cost function is,

$$
\begin{aligned}
& F_{i}\left(P_{i}\right)=F_{i}\left(P_{i}^{\mathrm{min}}\right)+s_{i 1} P_{i 1}+s_{i 2} P_{i 2}+s_{i 3} P_{i 3} \\
& 0 \leq P_{i x} \leq P_{i x}^{\mathrm{max}} ; x=1,2,3 \\
& P_{i}=P_{i}^{\mathrm{min}}+P_{i 1}+P_{i 2}+P_{i 3}
\end{aligned}
$$

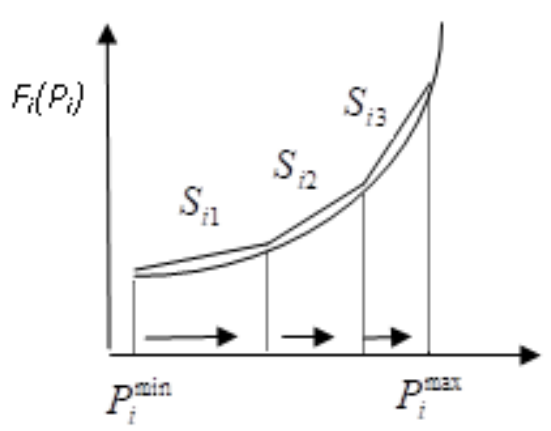

Fig.4. Linearized fuel cost curve

\subsection{LP Based SCOPF}

The Security Constrained Optimal Power Flow optimization problem using Linear Programming:
Linearized cost function,

$\min : F_{i}\left(P_{i}\right)=F_{i}\left(P_{i}^{\mathrm{min}}\right)+s_{i 1} P_{i 1}+s_{i 2} P_{i 2}+s_{i 3} P_{i 3}(15$

Equalityconstraint

$$
P_{\text {gen }}-P_{\text {load }}-P_{\text {loss }}=0 \text {; Power balanceequation }
$$

Inequalityconstraint,

$$
P_{i}^{\min } \leq P_{i} \leq P_{i}^{\max } ; \quad \text { Generator limits }
$$

Line flow limits (pre- contingency case)

$$
P_{m n}^{\min } \leq \sum_{i=1}^{n}\left[P T D F_{m n, i} \times P_{i}\right] \leq P_{m n}^{\max }
$$

Line flow limits (post - contingeng case)

$$
P_{m n}^{\min } \leq \sum_{i=1}^{n}\left[O T D F_{m n, i j} \times P_{i}\right] \leq P_{m n}^{\max }
$$

where $F_{i}$ is the cost function of generator $i, P_{i}$ is the real power output of generator $i, \mathrm{P}_{\mathrm{i}}^{\min }$ and $\mathrm{P}_{\mathrm{i}}^{\max }$ are the minimum and maximum are the generator $i$ limits, and $\mathrm{P}_{\mathrm{mn}}{ }^{\min }$ and $\mathrm{P}_{\mathrm{mn}}{ }^{\max }$ are minimum and maximum thermal limits of the line, respectively.

\subsection{LP Based Modified SCOPF}

The SCOPF with PR control, optimization problem incorporates the PR phase angle injection as part of the objective function and the optimization constraints. The SCOPF with PR control allows for the objective function to be further optimized, compared to the SCOPF, while obeying constraints that can now be modified by PR operation.

The objective function is given as,

$$
F_{i}\left(P_{i}\right)=F_{i}\left(P_{i}^{\mathrm{min}}\right)+s_{i 1} P_{i 1}+s_{i 2} P_{i 2}+s_{i 3} P_{i 3}+0 \phi_{x y}
$$

The equality constraint remains the same and the inequality constraints are as follows:

Line flow limits (pre-contingency case)

$P_{m n}^{\min } \leq \sum_{i=1}^{n}\left[\left(P T D F_{m n, i} \times P_{i}\right)-\left(P F R P T D F_{m n, i, \phi_{x y}} \times \phi_{x y}\right)\right] \leq P_{m n}^{\max }(21)$ Line flow limits (post-contingency case)

$$
P_{m n}^{\min } \leq \sum_{i=1}^{n}\left[\left(O T D F_{m n, l k} \times P_{i}\right)-\left(P F R P T D F_{m n, l k, \phi_{x y}} \times \phi_{x y}\right)\right] \leq P_{m n}^{\max }(22)
$$




\section{Results And Discussion}

A 6-bus system is used to produce results for this section. The network diagram is given in Fig.5 [4]. The test system has 11 Transmission lines. The resistance and reactance for the lines are obtained from the data set. The SCOPF code was programmed in MATLAB. The optimization problem is solved using the LINPROG function. All sensitivity factors are derived and calculated within the MATLAB using the $Y_{\text {bus }}$ and used as a subroutine in the main program.

Initially, a base-case result is produced by running a conventional OPF algorithm. We used, the operating cost and real power flow in the line as metrices for comparison to the SCOPF algorithm. The comparison results for operating costs and line flows are given in Table 1. A comparison between the line flows from the base case and precontingency case is in Table 2. It could be observed that in the base case three lines 2-4, 2-5, 3-5 have got overloaded in the attempt to optimize the problem. This overload can be relieved by adding line constraints to these three lines.

For the purpose of demonstration, a single line 23 outage is considered here. Without adding security constraints, three of the other lines $2-4,2-5,2-6$ have been overloaded due to outage of line 2-3. The SCOPF algorithm includes post-contingency constraints, i.e., Security constraints, which prevents additional power from being transmitted through these lines.

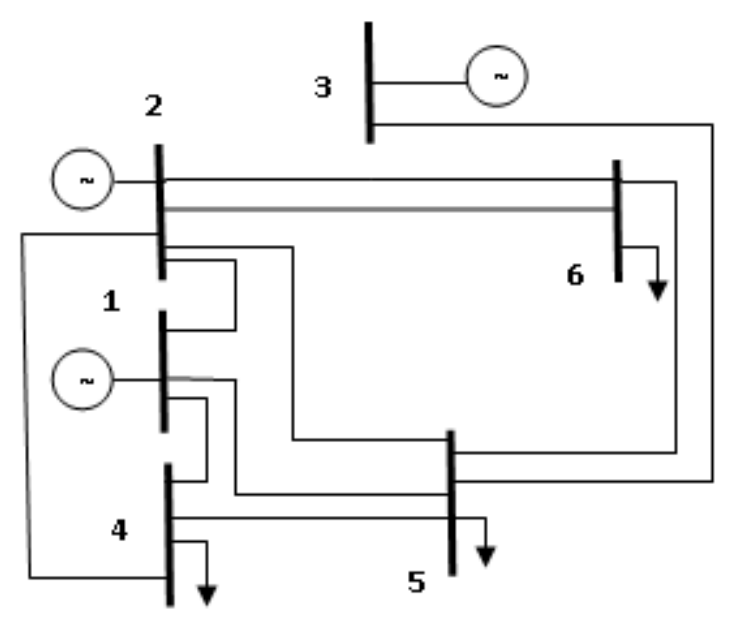

Fig.5. Topology of the 6-Bus Test System
A comparison between the line flows using DC power flow and $\mathrm{AC}$ power flow techniques are given in TABLE 4. It could be observed that the DC power flow has a closer value with the $\mathrm{AC}$ power flows. Though DC power flow analysis gives approximate results, it is non-iterative and solution could be obtained quickly without much computational effort.

The power router has been included in line 2-4. A phase angle adjustment of 2 degrees is done by the power router. The effect of power router on line flows and the fuel cost are given in TABLE 5. It could be observed that the power flow router minimizes the overall fuel cost and it also relieves the congestion in the line 2-4. The comparison of fuel costs has been given in Table 1. It is observed that the fuel cost is minimized by inclusion of power routers in the line.

Table 1

Comparison Of Operating Cost And Generation Schedules Between The OPF And SCOPF

Algorithms

\begin{tabular}{|c|c|c|c|c|}
\hline \multirow[t]{2}{*}{ Problem } & \multicolumn{3}{|c|}{ Generation Schedules } & \multirow{2}{*}{$\begin{array}{l}\text { Operati } \\
\text { ng Cos } \\
(\$ / \mathrm{hr})\end{array}$} \\
\hline & $\mathrm{P} 1$ & $\mathrm{P} 2$ & $\mathrm{P} 3$ & \\
\hline OPF & 100.3 & 61.61 & 54.99 & 3169.6 \\
\hline SCOPF & 100 & 56.79 & 60.07 & 3171.9 \\
\hline $\begin{array}{l}\text { MODIFIED } \\
\text { SCOPF }\end{array}$ & 100 & 59.40 & 57.4682 & 3170.5 \\
\hline
\end{tabular}

Table 2

Comparison Of Base Case And Pre-contingency Results

\begin{tabular}{l|l|l|l}
\hline \multirow{2}{*}{ Line } & \multirow{2}{*}{$\begin{array}{c}\text { Line Limit } \\
(\mathrm{MW})\end{array}$} & \multicolumn{2}{|c}{ Line Flows (MW) } \\
\cline { 3 - 4 } & & Base case & $\begin{array}{c}\text { Pre- } \\
\text { contingency } \\
\text { case }\end{array}$ \\
\hline $1-2$ & 30 & -0.7598 & 25.2988 \\
\hline $1-4$ & 50 & 27.6872 & 45.2988 \\
\hline $1-5$ & 40 & 20.4349 & 36.8659 \\
\hline $2-3$ & 20 & -7.1361 & 5.1414 \\
\hline $2-4$ & 40 & 56.8940 & 40.000 \\
\hline
\end{tabular}




\begin{tabular}{l|l|l|l}
\hline $2-5$ & 20 & 20.9414 & 20.000 \\
\hline $2-6$ & 30 & 21.0775 & 29.6059 \\
\hline $3-5$ & 20 & 31.0248 & 18.1332 \\
\hline $3-6$ & 60 & 59.9952 & 46.3582 \\
\hline $4-5$ & 20 & 1.4826 & 5.0000 \\
\hline $5-6$ & 20 & -6.8898 & -0.2627 \\
\hline
\end{tabular}

Table 3

Comparison of Line Flows with and Without Security Constraints

\begin{tabular}{c|c|c|c}
\hline \multirow{2}{*}{ Line } & \multirow{2}{*}{$\begin{array}{c}\text { Line } \\
\text { Limit } \\
(\mathrm{MW})\end{array}$} & $\begin{array}{c}\text { Line Flows (MW) } \\
\text { Security } \\
\text { Constraints }\end{array}$ & $\begin{array}{c}\text { With } \\
\text { Security } \\
\text { Constraints }\end{array}$ \\
\hline $1-2$ & 30 & 24.4929 & 25.3934 \\
\hline $1-4$ & 50 & 45.0009 & 45.3442 \\
\hline $1-5$ & 40 & 37.5613 & 36.8350 \\
\hline $2-3$ & 20 & 0.0000 & 0.0000 \\
\hline $2-4$ & 40 & 41.0159 & 39.9016 \\
\hline $2-5$ & 20 & 21.2327 & 19.9060 \\
\hline $2-6$ & 30 & 32.4577 & 29.4025 \\
\hline $3-5$ & 20 & 16.1156 & 18.2682 \\
\hline $3-6$ & 60 & 43.1180 & 46.5842 \\
\hline $4-5$ & 20 & 5.6706 & 4.9541 \\
\hline $5-6$ & 20 & 0.4058 & -0.3044 \\
\hline
\end{tabular}

Table 4

Comparison of dc power flows with the ac power flow solution

\begin{tabular}{c|l|l|l}
\hline Line & $\begin{array}{c}\text { Line Limit } \\
(\mathrm{MW})\end{array}$ & $\begin{array}{c}\text { DC Power } \\
\text { Flow(MW) }\end{array}$ & $\begin{array}{c}\text { AC Power } \\
\text { Flow(MW) }\end{array}$ \\
\hline $1-2$ & 30 & 25.3934 & 23.611 \\
\hline
\end{tabular}

\begin{tabular}{l|l|l|l}
\hline $1-4$ & 50 & 45.3442 & 42.050 \\
\hline $1-5$ & 40 & 36.8350 & 35.191 \\
\hline $2-3$ & 20 & 0.0000 & 0.000 \\
\hline $2-4$ & 40 & 39.9016 & 40.367 \\
\hline $2-5$ & 20 & 19.9060 & 18.720 \\
\hline $2-6$ & 30 & 29.4025 & 29.449 \\
\hline $3-5$ & 20 & 18.2682 & 20.584 \\
\hline $3-6$ & 60 & 46.5842 & 44.302 \\
\hline $4-5$ & 20 & 4.9541 & 4.444 \\
\hline $5-6$ & 20 & -0.3044 & 0.549 \\
\hline
\end{tabular}

Table 5

Line flows after including power routers

\begin{tabular}{l|l|l|l}
\hline Line & $\begin{array}{l}\text { Line Limit } \\
(\mathrm{MW})\end{array}$ & $\begin{array}{l}\text { DC Power } \\
\text { Flow(MW) }\end{array}$ & $\begin{array}{l}\text { AC Power } \\
\text { Flow(MW) }\end{array}$ \\
\hline $1-2$ & 30 & 24.4630 & 23.611 \\
\hline $1-4$ & 50 & 44.1298 & 42.050 \\
\hline $1-5$ & 40 & 33.3917 & 35.191 \\
\hline $2-3$ & 20 & -0.0001 & 0.000 \\
\hline $2-4$ & 40 & 39.3337 & 40.367 \\
\hline $2-5$ & 20 & 17.0831 & 18.720 \\
\hline $2-6$ & 30 & 21.7036 & 29.449 \\
\hline $3-5$ & 20 & 24.4632 & 20.584 \\
\hline $3-6$ & 60 & 55.7624 & 44.302 \\
\hline $4-5$ & 20 & 2.9789 & 4.444 \\
\hline $5-6$ & 20 & -2.6140 & 0.549 \\
\hline
\end{tabular}




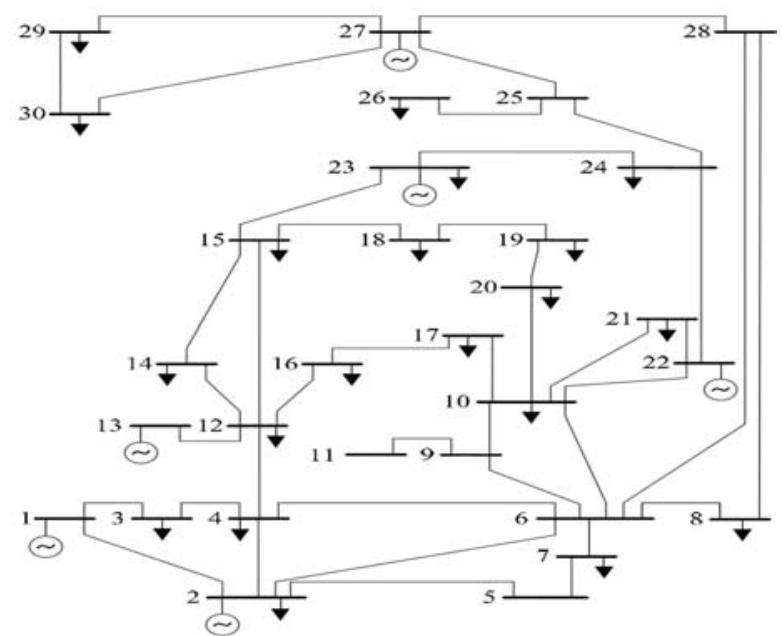

Fig.6. Topology of the 30-Bus Test System

The 30-bus IEEE Test case system seen in Fig.6, was used to produce the results. The given system consists of 41 lines and 6 Generators connected to bus $1,2,13,22,23$ and 27. In this system, Bus 1 is considered as the Slack bus. Only MW flow constraints are considered here (both in precontingency and post-contingency cases). By including power router in a line, the power flow in the line can be improved and also the overall fuel cost could be reduced. For the purpose of demonstration, an overload of line 2-4 is considered. This causes violation in that line. One power router is introduced in the line 2-4. The power router has been set to inject a phase angle of 2 degrees in the line.

The comparison of line flows with and without including PRs are given in Table 6.

The comparison of the Generation schedules and the operating cost of OPF, SCOPF and Modified SCOPF is given in Table 7. The results of OPF, SCOPF and Modified SCOPF show that by the inclusion of Power Routers in the line, the operating cost of the system can be minimized further.

Table 6

Comparison of line flows with and without power routers

\begin{tabular}{c|c|c|c}
\hline Line No. & $\begin{array}{c}\text { Line } \\
\text { Limits } \\
\text { (MW) }\end{array}$ & $\begin{array}{c}\text { Line flows } \\
\text { without } \\
\text { PR }\end{array}$ & $\begin{array}{c}\text { Line flows } \\
\text { with PR }\end{array}$ \\
\hline $1-2$ & 90 & 89.1333 & 81.0554 \\
\hline $1-3$ & 100 & 54.1919 & 64.4377 \\
\hline
\end{tabular}

\begin{tabular}{|c|c|c|c|}
\hline $2-4$ & 52 & 35.0016 & 50.9542 \\
\hline $3-4$ & 100 & 30.8341 & 41.6246 \\
\hline $2-5$ & 90 & 22.7755 & 32.2762 \\
\hline $2-6$ & 42 & 40.8863 & 0.0001 \\
\hline $4-6$ & 70 & 27.2577 & 54.3382 \\
\hline $5-7$ & 60 & 24.5968 & 34.9510 \\
\hline $6-7$ & 80 & 1.9678 & -7.8738 \\
\hline $6-8$ & 60 & 29.8465 & 29.8482 \\
\hline $6-9$ & 80 & 13.4638 & 11.7031 \\
\hline $6-10$ & 40 & 7.8796 & 6.9223 \\
\hline $9-11$ & 30 & 0 & 0.0000 \\
\hline $9-10$ & 50 & 14.3689 & 12.8596 \\
\hline $4-12$ & 70 & 15.6654 & 14.4858 \\
\hline $12-13$ & 40 & -18.0088 & -22.4333 \\
\hline $12-14$ & 30 & 7.5769 & 7.9688 \\
\hline $12-15$ & 30 & 14.7091 & 16.5623 \\
\hline $12-16$ & 30 & 6.3561 & 8.2787 \\
\hline $14-15$ & 30 & -0.1044 & 0.6035 \\
\hline $16-17$ & 30 & 1.9927 & 3.8486 \\
\hline $15-18$ & 30 & 7.9421 & 8.9914 \\
\hline $18-19$ & 30 & 3.9815 & 5.0337 \\
\hline $19-20$ & 50 & -7.8763 & -6.8195 \\
\hline $10-20$ & 30 & 10.2061 & 9.1911 \\
\hline $10-17$ & 30 & 1.7394 & -0.0591 \\
\hline $10-21$ & 40 & 6.1496 & 5.9354 \\
\hline $10-22$ & 30 & 0.6406 & 0.4991 \\
\hline $21-22$ & 70 & -15.4483 & -15.6670 \\
\hline
\end{tabular}




\begin{tabular}{c|c|c|c}
\hline $15-23$ & 30 & -3.6741 & -2.4960 \\
\hline $22-24$ & 30 & 4.8512 & 4.4406 \\
\hline $23-24$ & 30 & 4.7691 & 5.9415 \\
\hline $24-25$ & 30 & -1.8816 & -1.0111 \\
\hline $25-26$ & 30 & 5.0687 & 5.0687 \\
\hline $25-27$ & 30 & -6.2657 & -5.4303 \\
\hline $28-27$ & 50 & 7.0005 & 6.6295 \\
\hline $27-29$ & 90 & 7.7696 & 7.7696 \\
\hline $27-30$ & 30 & 8.8867 & 8.8867 \\
\hline $29-30$ & 100 & 4.6973 & 4.6973 \\
\hline $8-28$ & 30 & -3.0369 & -3.1329 \\
\hline $6-28$ & 50 & 10.7874 & 10.4683 \\
\hline
\end{tabular}

Table 7

Comparison Of Operating Cost And Generation Schedules Between The OPF And SCOPF Algorithms For 30-Bus System

\begin{tabular}{c|c|c|c}
\hline Method & OPF & SCOPF & $\begin{array}{c}\text { MODIFIED } \\
\text { SCOPF }\end{array}$ \\
\hline P1 & 126.0425 & 126.3385 & 127.4165 \\
\hline P2 & 40 & 296.281 & 329.747 \\
\hline P3 & 15 & 22.4333 & 48.0088 \\
\hline P4 & 15 & 18 & 18 \\
\hline P5 & 10 & 10 & 10 \\
\hline P6 & 12 & 12 & 12 \\
\hline $\begin{array}{c}\text { Operating } \\
\text { Cost } \\
(\$ / h r)\end{array}$ & 565.1593 & 573.1387 & 569.6367 \\
\hline \multicolumn{4}{|c}{}
\end{tabular}

\section{Conclusion}

An extension to the traditional SCOPF is made in this work by including Power routers in the power system. This further optimizes the system, thus maintaining the security requirements. The SCOPF is solved using Linear Programming technique and the line flows are calculated using DC Power Flow approach. Though the results obtained from the DC power flow are approximate values, this approach seems to be less time consuming and avoids the complex iterative procedure. The inclusion of power routers in a line relieves overloads in that particular line but it may sometimes lead to congestion of lines connected in parallel to the line with PRs.

\section{References:}

[1] B Stott and J.L Mannho, "Linear Programming for Power-System Network Security Applications", IEEE Trans On PAS, May/June, 1979, p 837.

[2] R. Podmore, "Economic Power Dispatch With Line Security Limits",IEEE Trans. On PAS, Jan/Feb 1974. p. 289

[3] B.F Wollenberg and W.O. Stadlin, "A RealTime Optimizer for Security Dispatch", IEEE Trans. On PAS, Jan/Feb 1974. p. 1640.

[4] A. J. Wood and B. F. Wollenberg, Power Generation, Operation, and Control. New York, NY, USA: Wiley, 2012.

[5] C W. Sanders and C.A Monroe, "A Algorithm for Real-Time Security Constrained Economic Dispatch", IEEE Trans. on Power Systems, Vol. 2, No 4, November, 1987, pp. 1068-1076.

[6] P. W. Sauer, "On the Formulation of Power Distribution Factors for Linear Load Flow Methods," IEEE Trans. Power Appar. Syst., vol. PAS-100, no. 2, pp. 764-770, 1981.

[7] Y. Ping, A. Sekar (2001). "A new approach to security-constrained optimal power flow analysis", Power Engineering Society Summer Meeting, 2001, vol. 3, pp. 1462-1467.

[8] D. S. Kirschen, "Power system security," Power Eng. J., vol. 16, no. 5, pp. 241-248, Oct. 2002.

[9] V. J. Mishra, and M. D. Khardenvis, "Contingency analysis of power system", in Proc. 2012 IEEE Students' Conf. on Electrical, Electronics and Computer Science (SCEECS), pp. 1-4, March 2012.

[10] Mithun Bhaskar M and Srinivas Muthyala, "Security Constrained Optimal Power flow - A comprehensive survey", in International Journal of Computer Applications, Volume 11, No.6, December 2010.

[11] J. Verboomen, D. Van Hertem, P. H. Schavemaker, W. L. Kling, and R. Belmans, "Phase shifting transformers: Principles and applications," in Proc. 2005 Int. Conf. Future Power Systems, 2005, p. 6.

[12] N. G. Hingorani, "Flexible AC transmission," Spectr. IEEE, vol. 30, no. 4, pp. $40-45$, Apr. 1993. 
[13] M. Noroozian, L. Angquist, M. Ghandhari, and G. Andersson, "Use of UPFC for optimal power flow control," IEEE Trans. Power Del., vol.12, no.4, pp. 1629-1634, Oct. 1997.

[14] R. P. Kandula, A. Iyer, R. Moghe, J. E. Hernandez, and D. Divan, "Power flow controller for meshed systems with a fractionally rated BTB converter," in Proc. 2012 IEEE Energy Conversion Congr. Expo. (ECCE), Sep. , pp. 4053-4060.

[15] D. Das, D. M. Divan, and R. G. Harley, "Power flow control in networks using controllable network transformers," IEEE Trans. Power Electron., vol. 25, no. 7, pp. 1753-1760, 2010.

[16] D. Divan and J. Sastry, "Voltage synthesis using dual virtual quadrature sources-A new concept in AC power conversion," in Proc. IEEE Power Electron. Spec. Conf., 2007 (PESC), Jun., pp. 2678-2684. 